\title{
INFORMATION SYSTEMS FOR CRISIS MANAGEMENT - CURRENT APPLICATIONS AND FUTURE DIRECTIONS
}

The management of crisis situations is undergoing rapid changes due to advances of Information Technology. We discuss the role of information systems in supporting decision making processes in crisis management. The crisis management poses particular challenges for information systems - it is characterized by dynamic, complex environments involving many actors in often very unique and extreme situations. We review the roles, benefits and challenges of information systems' application to different phases of the crisis management. We argue that for the different phases of the crisis management, the different types of information systems varying in the aspects such as use scenarios, user requirements, critical technologies, etc. are suitable. Finally, we outline the directions for future research and applications for the field of the support systems for the emergency management.

\section{Introduction}

After over three decades of application of computer based information systems to the crisis management, these systems are getting wider acceptance by the community of the emergency managers. The need for Emergency Management Information Systems (EMIS) (for more see [12]) supporting the decision makers working under pressure and facing dynamically changing environments has been recognized by both practitioners and researchers. Disaster, crisis, catastrophe and emergency management are very often used synonymously and sometimes with slight differences, especially with practitioners and researchers. We use the term crisis management to emphasize that we are not concerned about small scale emergencies such as traffic accidents or building fires, but our focus is on disasters and catastrophes, no matter if natural or man-made. We would like to keep the Emergency Management Information Systems (EMIS) as the term used in the field for all of the information systems used for the crisis response and management support. The United Nations defined disaster as a serious disruption of the functioning of a society, and catastrophes refer to disasters causing such widespread human, material, or environmental losses that exceed the ability of the affected part of society to cope adequately using only its own resources. Both disasters and catastrophes create crisis situations.

\section{Information Systems in Crisis Management}

As defined by Simak [11], an information system is a system able to deliver information for immediate or later action. Concretely, it is a set of procedures, tasks, activities, people and technologies. An information system has four specific functions:

- data collection,

- data storage,

- data processing and analysis,

- data transfer and distribution.

Crisis management is one of the most challenging management tasks possible to imagine - combination of time pressure on decision makers, rapidly changing environment, uniqueness of each crisis situation, and high cost of decisions, often involving many human lives and large financial consequences makes crisis management a very difficult task. A general role of information systems in crisis management is to help decision making process information and make right decisions.

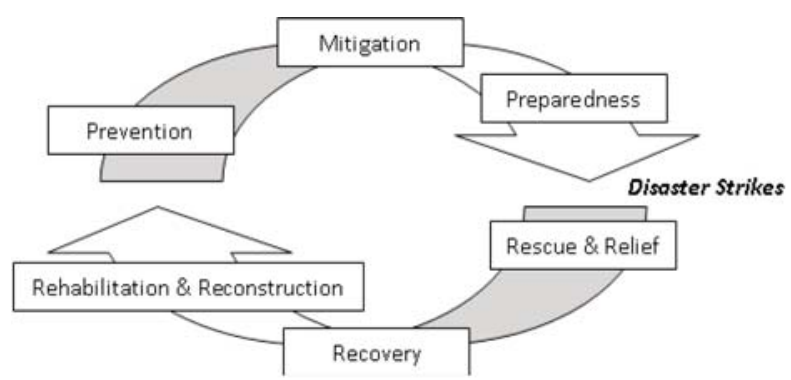

Fig. 1 Phases of disaster management cycle

\footnotetext{
* Jozef Ristvej ${ }^{1}$, Adam Zagorecki ${ }^{2}$

${ }^{1}$ Department of Crisis Management, University of Zilina, Slovakia, Assistant Professor, E-mail: Jozef.Ristvej@fsi.uniza.sk,

${ }^{2}$ Defence Academy of the United Kingdom, Cranfield University, Shrivenham, United Kingdom
} 
The literature within the crisis management typically identifies four to eight phases of the disaster management process, and presents them as a cycle [13]. The six phase cycle of disaster management cycle as presented in Fig. 1 by Menon and Sahay [9] is the most common one and will serve as the basis of our discussion.

Information systems can be a powerful tool that can support decision making process in all phases of the disaster management cycle. Historically, the main focus was on the response phase. With the paradigm shift, the role of crisis managers has immensely expanded and the focus is spread among all phases with emphasis on prevention.

We start by reviewing the role of EMIS for the different phases of the cycle. We argue that from the perspective of an EMIS development, the six phases of the disaster management cycle ask for different types of EMIS tools that differ in terms of:

- problems the system is intended to address,

- audience for which they are intended,

- importance of ability to provide real-time data,

- ability to accommodate multiple actors (especially multiple organizations),

- sophistication of used technologies,

- data presentation to the user.

In the remaining part of the paper we will discuss applications and development of EMIS in context of specific phases of the disaster management cycle with support of definition of each phase according to World Health Organization [14].

\section{Prevention and Mitigation}

Prevention is intended to eliminate possibility of disaster occurrence or, in vast majority of cases, to reduce the probability of disaster occurrence. Mitigation is intended to reduce the consequences of unavoidable disasters. Prevention and mitigation measures include building codes, vulnerability analyses, zoning and land use management, preventive health care, etc. their effectiveness depends on the availability of information on hazards, risks, appropriate measures in national and regional development.

The EMIS for prevention and mitigation focus mostly on two aspects: (1) collection relevant data that would be used for risk assessment and strategies to prevent them and/or mitigate their effects, and (2) providing tools for this data analysis that would enable to perform vulnerability analysis, define best policies, etc. The former aspect is concerned more on creating infrastructure for data collection and storage. It is mostly focused on creating repositories of documents (emergency plans, historical reports, etc.), maps (with use of GIS systems), historical measurements (for example weather information, river gage readings, medical records, etc.) and providing tools to access to this information by various actors and organizations. This type of systems uses well-established technologies based on databases, document processing and World Wide Web (WWW). From the technical perspective they do not differ from typical information systems for other domains - they are intended to be used on daily bases by domain experts who are responsible for planning and policy making, not the actual responders during crisis situations.

The later aspect is focused on systems that support analysis of risks and vulnerability. In this area special emphasis should be put on advanced methods for data analysis - machine learning, decision analysis, intelligent systems. These disciplines are experiencing rapid progress and are capable of delivering methods and solutions that can address real-life problems. The development of intelligent tools requires interdisciplinary approach where researchers, the emergency managers and domain experts should work closely to develop tools that address specific problems. It is a relatively immature area that requires significant research component, however is clearly becoming the mainstream for data analysis and decision making in the future.

\section{Preparedness}

The goal of preparedness programs is to achieve a satisfactory level of readiness to respond to any crisis situation through measures that strengthen the technical and managerial capacity of governments, organizations, and communities. These measures can be described as logistical readiness to respond to disasters. Readiness can be improved by developing mechanisms and procedures, rehearsals, long-term and short-term strategies, tactics, public education, and implementing early warning systems. Preparedness can also take the form of ensuring strategic reserves of food, resources, medicines, and others. Preparedness measures include emergency training and exercises, communications systems, evacuations plans, resource inventories and contact lists, mutual aid agreements, and public information/education.

The preparedness encapsulates a wide range of actions and approaches that differ in their nature. Therefore, EMIS for the preparedness phase can cover a wide range of applications and requirements on the information systems. We briefly discuss main types of EMIS that support the preparedness phase.

- Document and data sharing tools - the information technology enabled unprecedented ease of storage and access to large variety of data. During the preparedness phase it is possible to collect comprehensive data related to threats and response to them: databases of available resources (on local and national level), qualifications and contact addresses of trained personnel, hazard databases (for example localization and amounts of hazardous materials), factsheets on hazardous materials, etc. This data should be easily accessed when needed and kept up to date (which is often a practical challenge). In terms of technology, this type of systems utilizes well-established database technology and WWW user interfaces. Often trade offs between ease of use and protection from unauthorized access is an important issue because of the fact that some of this data can be proprietary (e.g. water or electricity providers) or pose a security threat. For example, a database of hazardous materials or detailed plans of power grid can be potentially a very dangerous in hands of terrorists. 
- Geographical Information Systems (GIS) - even though the GIS can fall into the category above, the traditional importance and practical value of maps in the emergency management makes it worth discussing them separately. The GIS concept is far more than just a digital version of traditional maps. It allows for real time updating, publishing, and advanced spatial analysis of data. The visual representation of data is especially suitable for humans and can enhance decision making processes.

- Early warning systems - this type of EMIS is intended to provide an advance warning prior to a disaster. They are threat specific and more typical for natural disasters: e.g. tsunami warning systems or extreme weather warning systems. They may include warning dissemination systems. They may range from simple sensing devices, often networked, to complex predictive models based on simulation and physical models. A common element is their end user - a human decision maker who is responsible for making a decision for issuing the warning.

- Training applications - a proven practical use of the EMIS are tools for training and exercise generation. The computer-based support systems can use the data collected for the other purposes such risk assessment to generate realistic exercise scenarios. One of the proposed uses of the virtual reality is use as a training tool for the responders to familiarize them with terrain and infrastructure characteristics of the area where the response will take place. In particular it can be useful for the response in the areas with which the responders are not familiar with - in the context large disasters involving international rescue teams.

- Decision support systems - a wide category of EMIS that are intended to support decision-makers by providing real-time, or close to real-time, data on various aspects of disaster management - e.g. resource management or situational awareness. Even though these systems are primarily intended for everyday use in non-disaster environment, their application can be extended to the crisis situations. One of the key strengths of these systems is users' familiarity with them gained through everyday use. Other specialization is the dashboard system that provides realtime data visualization by means of key performance indicators - abstract measures that provide rapid assessment of the evolving situation. A dashboard system to monitor hospital system with available number of beds, categorized by type of units and specialization, can be a good example.

By no means is the list above complete - the preparedness phase depends heavily on the nature of disasters for which it is intended. Particular regions can greatly differ in the risks (for example: earthquakes, tsunamis or major river floods are characteristic to some regions and are not a viable threat for others). So are the preparedness measures - they should be always customized toward local threats, community characteristics, available resources, etc.

\section{Rescue and Relief}

During crisis situations the initial response is by the government and professional organizations. Humanitarian agencies join shortly after. To respond effectively, these organizations must have experienced leaders, trained personnel, adequate transport and logistic support, appropriate communications, and guidelines for working in emergencies. A real challenge is coordination of these, often different in structure and operation, organizations. The aim of the response is to provide immediate assistance to sustain life, support recovery and providing other necessary services. Such assistance may range from providing specific but limited aid, such as transport, shelters, and food, to establishing semi-permanent settlements. It also may involve initial repairs to damaged infrastructure. The focus in the response phase is on meeting the basic needs of the affected population until more permanent and sustainable solutions are feasible.

During the rescue and immediate relief phases, the EMIS can take on critical roles of providing communication and rapid data sharing. The decision support systems that are used during everyday emergency management can provide their services during and after the disaster strikes. However, the after disaster response creates a need for different type of data, communication and decision support that is needed during everyday routine. In particular, the disaster response calls for extensive multi-organizational communication far beyond the level needed during non-crisis times. Information technology, in particular communication technology can offer enhanced means of communication.

The multi-organizational data sharing is probably the key aspect of the response that can be addressed by means of computer-based information systems. The Sahana [14] project is such an example. Sahana was initiated during the response to 2004 Indian Ocean tsunami and since then used in many major disaster response efforts throughout the world. Sahana intends to facilitate communication between response organizations and individuals on number of aspects, including, but not limited to: missing person registry, response organization registry, request management, personnel tracking, etc. One notable tool is the Situational Awareness module - a tool where individuals can place information, including photographs to create the common operating picture.

\section{Recovery}

As the crisis is brought "under control", the affected population is capable of undertaking a growing number of activities aimed at restoring their normal lives and reconstruct the infrastructure. The recovery period creates a unique opportunity to improve on prevention and preparedness. Ideally, there should be a smooth transition from recovery to on-going development. The recovery activities, both short and long term, include returning vital life-support systems to minimum operating standards, temporary housing, public information, health and safety education, etc. Information resources and services include data collection related to rebuilding, and documentation of lessons learned.

The recovery phase is about actions to restore normal operations. The EMIS used in this phase are a mixture of the EMIS for rescue and relief and the systems that support reconstruction and even the prevention and mitigation phases. Therefore, we believe 
they have no distinctive features/requirements that would validate discussing them as an individual category.

\section{Rehabilitation and Reconstruction}

In the rehabilitation and reconstruction phase, considerations of risk reduction should form the foundations for all activities. The rehabilitation and the reconstruction phases are in essence about learning lessons from the disaster and the response. No matter how prepared the community was, in practice, it is always possible to substantially improve the disaster response. The first step is to collect data to gain understanding of the disaster and the response to it. EMIS can be useful in this phase. Moreover, the lessons learned may provide insight into use of EMIS in the response process - it is especially valuable because the EMIS are relatively new to the field and we believe that the concept is still in the phase of proving its value and defining directions for further development.

\section{Directions for Future}

In the previous sections we discussed the state-of-the-art in terms of providing support for crisis situations. We discussed the roles and requirements on EMIS for all the phases of the disaster response. Now, based on that discussion we would like to indicate some directions for development of future EMIS, they are:

- Personal computing - the Internet becomes increasingly popular, with over 1.6 billion as of Nov 2010, [3]. Internet affects how we communicate, access and share information. In the recent years popularity of cell phones, and in particular smart phones created new ways how people can access and share information even faster than ever before. Development of tools for personal computing especially in the area of the communication, geo-location (most of modern smart phones include GPS functionality), instant image sharing and access (build-in cameras), have a great potential to transform disaster management.

- Growing knowledge bases - more and more organizations are organizing their data, document processing, and other processes with use of the IT. During this process data and knowledge bases are created, enabling the EMIS to access more and more data, making certain aspects of the disaster response possible. In terms of data sharing, the sharing standards such as XML and web services are making interoperability much easier than in the past.

- Sensing technologies - the development of new sensing technologies, resulted in popularity and lowering the cost of different type of sensors, ranging from regular cameras to specialized types such as vibration sensors. The current trend is to use network-enabled sensors that can stream the real-time data to computer networks. The low cost of individual sensors enabled sensor networks - large numbers of interconnected sensors intended to improve situational awareness through sensing at multiple points at the same time.

- Simulation - increasing computational power lead to development of more sophisticated modeling and simulation techniques.
Traditionally the researchers in the field of emergency management had two methods of investigation: theory and experiment. The computers enabled to address the problems that are too complex to understand theoretically or through the natural experiment [7]. Bezivin and Gerbe [2] define a model as a simplification of a system built with an intended goal in mind. The model should be able to answer questions in place of the actual system. A model is an abstract description of a system or its part written in a language. This language should have a well-defined syntax and interpretation which is suitable for automated interpretation by a computer [6]. Banks [1] defines a simulation as an imitation of an operation of a real-world process or system over time. To prepare and make a simulation we need to have a model. Simulation is a valuable problem-solving methodology for many real-world problems that are characterized by complexity and uncertainty. Simulation can be used to ask "what if" questions about the real system, and investigate the consequences of changing the design of real systems. Both existing and conceptual systems can be modeled with simulation.

- Intelligent systems - with the development of artificial intelligence, and related techniques for analyzing data, and drawing automated conclusions (machine learning, data mining, etc.), the computer-based tools become able to address increasingly complex problems. With many successful commercial tools based on these techniques (hardware diagnosis, credit valuation, customized user interfaces, etc.) it should be expected that they will slowly get recognition and applications in EMIS. Examples of applications in the crisis management include disease outbreak detection, evacuation modeling.

- Dashboard systems - importance of providing intuitive visualization of data, especially in the context of a rapidly developing crisis, is of critical importance. A dashboard system that is intended to provide situational awareness based on high-level, real-time measures has a potential to significantly enhance the emergency manager's toolbox. Especially, with increasing availability of digital databases and the development of sensing technologies, the dashboard systems can in future become a mainstream, or at least a significant class of EMIS.

A computer-based system for emergency management should:

- combine problem-specific tools into a comprehensive and integrated set of tools,

- include up to date and relevant data,

- be able to store data, archive it and produce reports based on this data,

- be capable of making anticipations,

- provide secure but easy and reliable access,

- have intuitive human computer interface.

Discussing the EMIS would not be complete without the discussion of challenges and problems the EMIS are facing. Probably the most obvious is the cost of implementation of the EMIS and their relative value to the benefits they bring. Clearly, there are systems that are beneficial from both the user's perspective (enhance human decision-making) and they are cost-effective in terms of reduction of already allocated resources. One of the facts that are surprisingly often ignored is the maintenance the system - often 
the response organizations budget for introduction of a new system, but do not expect costs for the maintenance of the system, in particular keeping the database up to date.

\section{Conclusion}

We discussed the role of information systems in the crisis management, the current applications and future directions. The information systems for the disaster management reflect the complexity and diversity of the challenges faced at the different phases of the disaster response. We argued that the problem of development and application of EMIS should be considered in the context of phases of the disaster management, in order to address the diversity of EMIS.

The potential benefits and importance of information systems for crisis management are gaining recognition by practitioners at the all phases of the disaster management. We argue that the EMIS concept is a success measured by practical applications. There are numerous examples of EMIS that are used in a daily practice. We observe development of a wide variety of systems that contribute to the overall set of solutions. The EMIS are becoming more complex, addressing more challenging problems. There is a very active interest of the research community in proposing new ideas that, at least some of them, should soon result in new applied systems.

We believe that the future of the information systems for disaster management lies with integrated information systems that build upon a set of problem-specific tools. We view it as a gradual process where advances in modeling, simulation, data collection, etc. will lead to more complex and interdependent tools that ultimately will provide comprehensive solutions to the overall emergency management at all the phases. Until it happens, there will be a slow process of improving existing tools and developing new techniques, often based on the emerging new technologies.

\section{Acknowledgments}

Supported from the Project VEGA Nr. 1/0797/10, Komplexne modelovanie rizik ohrozujucich bezpecnost miest / Complex Modeling of Risks as a Security Threats for Cities.

\section{References}

[1] BANKS, J.: Introduction to Simulation, Proc. of the $31^{\text {st }}$ conference Winter Simulation Conference Archive on Winter Simulation: Simulation-a Bridge to the Future-Vol. 1, Phoenix, Arizona, 1999.

[2] BEZIVIN, J., GERBE, O.: Towards a Precise Definition of the OMG/MDA Framework, ASE'01, 2001.

[3] CIA, https://www.cia.gov/library/publications/the-world-factbook/rankorder/2153rank.html (retrieved 11/22/10), 2010.

[4] COMFORT, L.K., et al.: Designing Resilience - Preparing for Extreme Events. Pittsburgh : University of Pittsburgh Press, 2010.

[5] KAMPOVA, K. The Concept of Social Risks Perception. In: Risk analysis VII. Southampton: WIT Press, 2010, pp. PI-127-235.

[6] KLEPPE, A., et. al: MDA Explained. The Model Driven Architecture: Practice and Promise, Addison-Wesley, 2003.

[7] KUPERS, R.: What Organizational Leaders Should Know about the New Science of Complexity, Complexity, Vol. 6, No. 1, pp. 14-19, 2001.

[8] LOVECEK, T., et al: Critical Infrastructure Protection Systems Effectiveness Evaluation, J. of Homeland Security and Emergency Managemen, Vol. 7, No. 1, Article 34, ISSN: 1547-7355. DOI: 10.2202/1547-7355.1613 Available at: http://www.bepress.com/ jhsem/vol7/iss 1/34, 2010.

[9] MENON, N.V.C., SAHAY, R.: Role of Geoinformatics for Disaster Risk Management, available at: http://www.gisdevelopment.net/ magazine/years/2006/oct/26_1.htm, 2006

[10] SAHANA: Sahana Software, available at: http://www.sahanafoundation.org/ (retrieved 11/22/10), 2009.

[11] SIMAK, L.: Crisis Management in Public Administration (in Slovak), Zilina : FSI ZU, 2001, p. 243, ISBN 80-88829-13-5, 2001.

[12] TUROFF, M., CHUMER, M., VAN DE WALLE, B., YAO, X. (2004) The Design of a Dynamic Emergency Response Management Information System (DERMIS), J. of Information Technology Theory and Application (JITTA), Vol. 5, No. 4, pp. 1-36.

[13] TUROFF, M., HILTZ, S. R., WHITE, C., PLOTNICK, L., HENDELA, A., XIANG, Y.: The Past as the Future of Emergency Preparedness and Management, J. of Information Systems for Crisis Response and Management, Vol. 1, No. 1, pp. 12-28, 2009.

[14] WHO: Environmental Health in Emergencies and Disasters: a Practical Guide, available at: http://www.who.int/water_sanitation_health/hygiene/emergencies/ emergencies2002/en/ (retrieved 11/12/10), 2003.

[15] ZWASS, V.: Foundations of Information Systems, Boston : Irwin/McGraw-Hill, 1998. 